\title{
A zigzag model for laminated composite beams
}

\author{
Hemendra Arya *,1, R.P. Shimpi, N.K. Naik \\ Department of Aerospace Engineering, Indian Institute of Technology, Powai, Mumbai 400 076, India
}

\begin{abstract}
In the present work, a zigzag model for symmetric laminated beam is developed. This model uses a sine term to represent the nonlinear displacement field across the thickness as compared to a third order polynomial term in conventional theories. Transverse shear stress and strain are represented by a cosine term as compared to parabolic term. This model satisfies displacement and transverse shear stress continuity at the interface. Zero transverse shear stress boundary condition at the top and bottom of the beam are also satisfied. The numerical results indicates that the present model predicts very accurate results for displacement and stresses for symmetric cross-ply laminated beam, even for small length to thickness ratio. The results are also compared with a simplified theory of same class.
\end{abstract}

Keywords: Zigzag model; Composite laminated beam; Higher order shear deformation theory; Trigonometric shear deformation theory

\section{Introduction}

High in-plane strength and low density makes the polymer-matrix composites a promising candidate for high performance structures. Transverse shear deformation is a major issue in the analysis of these materials due to low value of shear modulus as compared to conventional materials. A lot of work has been done to estimate it correctly and efficiently for design purposes. Classical laminated beam theory does not model transverse shear deformation and is only appropriate for beams of high aspect ratio where it is not prominent.

Lo et al. $[1,2]$ proposed a higher order displacement model consisting of polynomial in thickness direction for analysis of composite plates. This kind of displacement model has been widely used with modifications to satisfy transverse shear stress boundary condition at the top and bottom of the plate [3,4]. In these models the displacement and its slope with respect to the thickness direction is continuous. This property of the displacement field leads to discontinuous transverse shear stress, and is also not able to model zigzag nature of the displacement field in thick laminated composite structures. These are also known as Equivalent Single Layer (ESL) theories.
In the later developments the displacement models are presented, which are layer dependent and are able to maintain in-plane displacement and shear stress continuity at the interface. In many of these models, number of variables are independent of number of layers $[5,6]$. In the present work also, a displacement model is presented in which the number of variables are independent of layers. The displacement field consists of a trigonometric sine term to represent the non-linear variation across the thickness in addition to classical beam theory terms. In conventional theories, a cubic term is used to represent this. The sine term in in-plane displacement leads to a cosine variation in transverse shear stress as compared to parabolic variation in conventional theories. A simplified ESL model of a similar class is also presented and results are compared. Stein [7] has used a similar plate theory for the post buckling analysis of plate, his theory does not satisfy the transverse shear stress condition at top and bottom of the plate. Recently, Shimpi and Ghugal [8] have used trigonometric terms in the displacement field for the analysis of two layer composite plate. Based on this theory, a finite element model was developed by Shimpi and Ainapure [9].

\section{Displacement field}

A composite laminate of $N$ laminae is shown in Fig. 1. $x$-axis is at the center of laminate and the $z$-axis is perpendicular to it. The displacement field at any point $(x, z)$ inside the laminate is given below: 


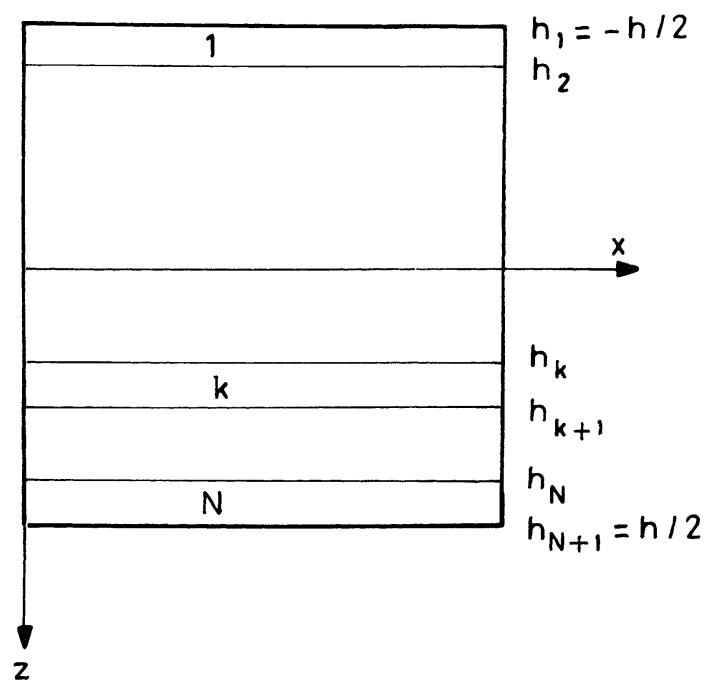

Fig. 1. Composite laminate geometry.

$u^{k}=u_{0}-z \frac{\partial w_{0}}{\partial x}+\left(A^{k}+z B^{k}+\sin \frac{\pi z}{h}\right) \eta_{x}$,

$w=w_{0}$,

where $u^{k}$ is in-plane displacement in $k$ th layer, $w_{0}$ is transverse displacement in $z$-direction, $\eta_{x}$ is higher order term, $h$ is the total thickness of the laminated beam and

$$
\begin{aligned}
A^{k}= & -\frac{\pi}{h} \sum_{n=2}^{k} h_{n}\left[\left(\cos \frac{\pi h_{n}}{h}\left(\frac{G_{x z}^{n-1}}{G_{x z}^{n}}-1\right)\right.\right. \\
& \left.+\sum_{p=2}^{n-1} \cos \frac{\pi h_{p}}{h} \frac{G_{x z}^{p}}{G_{x z}^{n}}\left(\frac{G_{x z}^{p-1}}{G_{x z}^{p}}-1\right)\right) \\
& -\left(\cos \frac{\pi h_{n-1}}{h}\left(\frac{G_{x z}^{n-2}}{G_{x z}^{n-1}}-1\right)\right. \\
& \left.\left.+\sum_{p=2}^{n-2} \cos \frac{\pi h_{p}}{h} \frac{G_{x z}^{p}}{G_{x z}^{n-1}}\left(\frac{G_{x z}^{p-1}}{G_{x z}^{p}}-1\right)\right)\right] \\
B^{k}= & \frac{\pi}{h}\left[\cos \frac{\pi h_{k}}{h}\left(\frac{G_{x z}^{k-1}}{G_{x z}^{k}}-1\right)\right. \\
& \left.+\sum_{m=2}^{k-1} \cos \frac{\pi h_{m}}{h} \frac{G_{x z}^{m}}{G_{x z}^{k}}\left(\frac{G_{x z}^{m-1}}{G_{x z}^{m}}-1\right)\right] .
\end{aligned}
$$

It may be noted that the displacement field is dependent on only three variables $u_{0}, w_{0}$ and $\eta_{x}$. The displacement field is different in different layers and is governed by the coefficient $A^{k}$ and $B^{k}$, which are dependent on layer material and geometry. $A^{k}$ and $B^{k}$ are zero for $N=1$ (single layer). Here $G_{x z}^{n-1}$ is transverse shear modulus and superscript is layer number.

The in-plane and transverse shear strains are:

$\epsilon_{x}^{k}=\frac{\partial u_{0}}{\partial x}-z \frac{\partial^{2} w_{0}}{\partial x^{2}}+\left(A^{k}+z B^{k}+\sin \frac{\pi z}{h}\right) \frac{\partial \eta_{x}}{\partial x}$, $\gamma_{x z}^{k}=\left(B^{k}+\frac{\pi}{h} \cos \frac{\pi z}{h}\right) \eta_{x}$

The governing equations associated with the final displacement functions can be derived using the virtual work principle. These variationally consistent equations are as follows:

$$
\begin{aligned}
& \delta u_{0}: \frac{\partial N_{x}}{\partial x}=0 \\
& \delta w_{0}: \frac{\partial^{2} M_{x}}{\partial x^{2}}-q=0 \\
& \delta \eta_{x}: \frac{\partial N_{A x}}{\partial x}+\frac{\partial M_{B x}}{\partial x}+\frac{\partial N_{S x}}{\partial x}-T_{B x}-\frac{\pi}{h} T_{c x}=0
\end{aligned}
$$

where $q$ is the distributed transverse load, and the resultant forces and moments are defined as follows:

$$
\begin{aligned}
& \left(N_{x}, N_{A x}, N_{S x}, M_{x}, M_{B x}\right) \\
& \quad=\sum_{k=1}^{N} \int_{h_{k}}^{h_{k+1}} \sigma_{x}^{k}\left(1, A_{k}, \sin \frac{\pi z}{h}, z, z B_{k}\right) \mathrm{d} z \\
& \left(T_{B x}, T_{c x}\right)=\sum_{k=1}^{N} \int_{h_{k}}^{h_{k+1}} \tau_{x z}^{k}\left(B_{k}, \cos \frac{\pi z}{h}\right) \mathrm{d} z .
\end{aligned}
$$

The essential and natural boundary conditions obtained are listed below:

$\begin{array}{ll}\text { Natural boundary } & \text { Essential boundary } \\ \text { conditions } & \text { conditions } \\ N_{x} & u_{0} \\ M_{x} & \partial w_{0} / \partial x \\ \partial M_{x} / \partial x & w_{0} \\ \left(N_{S x}+M_{B x}+N_{A x}\right) & \eta_{x}\end{array}$

\section{Numerical example and results}

The efficacy of the present zigzag displacement model further referred as TSDT-ZZ, is shown by comparing the results with the exact solution. The example chosen here is a symmetric three layer beam for which exact results are available in the literature [10]. It may be noted that though the results are available for cylindrical bending they are still good for comparison. The boundary conditions of the chosen beam at two ends are: $N_{x}=0, M_{x}=0, w_{0}=0$ and $N_{S x}+M_{B x}+N_{A x}=0$. All the layers are of equal thickness. The material properties of the layers are [10]:

$$
\begin{aligned}
& E_{1}=0.25 \times 10^{8} \mathrm{psi}, \\
& E_{2}=0.1 \times 10^{7} \mathrm{psi}, \\
& G_{13}=0.5 \times 10^{6} \mathrm{psi}, \\
& G_{23}=0.2 \times 10^{6} \mathrm{psi} .
\end{aligned}
$$


The following displacement function satisfies the simply supported boundary conditions mentioned earlier:

$u_{0}=\sum_{i=1}^{\infty} U_{i} \cos \alpha x$

$w_{0}=\sum_{i=1}^{\infty} W_{i} \sin \alpha x$,

$\eta_{x}=\sum_{i=1}^{\infty} \eta_{x i} \cos \alpha x$

The distributed load $q$ on the top of the beam can be expressed as

$q=\sum_{i=1}^{\infty} q_{i} \sin \alpha x$,

where $\alpha=i \pi / l$, and $l$ is the length of the beam. For sinusoidal load $i=1$.

The results are also compared with a ESL theory of similar class and it is further referred as TSDT-ESL, the displacement field is:

$u=u_{0}-z \frac{\partial w_{0}}{\partial x}+\sin \frac{\pi z}{h} \eta_{x}$

$w=w_{0}$.

It may be noted that in the above displacement function the derivative with respect to thickness is continuous.

All the calculated values are non-dimensionalised as given below:

$\bar{\sigma}_{x}=\frac{\sigma_{x}(l / 2, z)}{q}, \quad \bar{\tau}_{x z}=\frac{\tau_{x z}(0, z)}{q}, \quad \bar{u}=\frac{E_{2} u(0, z)}{h q}$,

$\bar{w}=\frac{100 E_{2} h^{3} w(l / 2,0)}{q l^{4}}$.

In Fig. 2 the variation of transverse central displacement $\bar{w}$ with different aspect ratios is shown. Results for TSDT-ZZ and TSDT-ESL shows good agreement with the exact results. All the exact results are retrieved from the figures available from literature. As expected the results obtained by TSDT-ZZ and TSDTESL are on lower side of exact results.

In Fig. 3 in-plane displacement $\bar{u}$ obtained by using TSDT-ZZ and TSDT-ESL are compared with the exact results. TSDT-ZZ is able to capture the kink observed in exact results. It may be noted that in exact results the inplane displacement is not zero at $z=0$ due to compressibility effects which are prominent in thick laminates. In TSDT-ZZ and TSDT-ESL this is not modeled and hence $\bar{u}$ is zero at $z=0$. In TSDT-ESL a single function is used to represent the variation of in-plane displacement across the thickness and its derivative with respect to thickness co-ordinate is also continuous. This is the limitation in TSDT-ESL which restricts to model the sudden change in direction of in-plane displacement occurring due to large variation in material properties

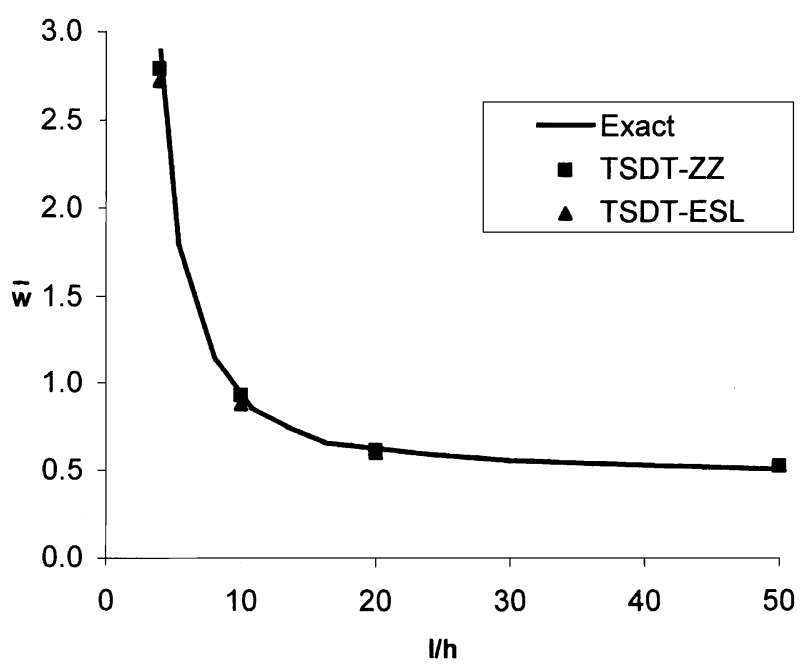

Fig. 2. Variation of central transverse displacement $\bar{w}$ with different aspect ratio $l / h$.

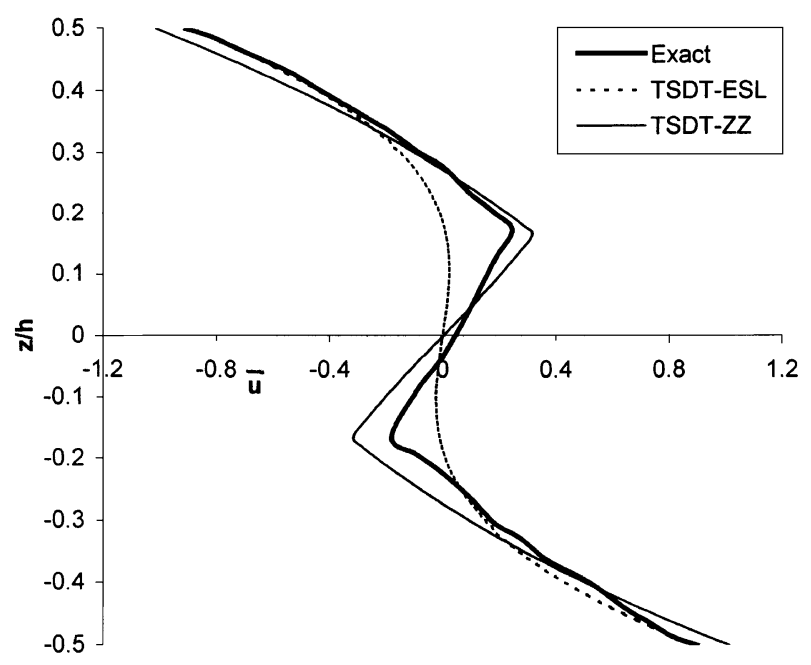

Fig. 3. Comparison of in-plane dispalcement $\bar{u}$ with exact results.

from layer to layer. The zigzag displacement model can take care of this and for thick laminates it becomes necessary to use such models. The curviness in the exact results has come because of interpolation while retrieving the data.

In Fig. 4 in-plane stress $\bar{\sigma}_{x}$, obtained using TSDT-ZZ and TSDT-ESL is compared with exact results. The trend shown by TSDT-ZZ is closer to the exact results as compared to TSDT-ESL. Peak stress prediction using TSDT-ZZ is on higher side as compared to exact results. In-plane stress $\bar{\sigma}_{x}$ is not zero at $z=0$ due to compressibility effects and it is not captured in the present displacement functions.

Transverse shear stress $\bar{\tau}_{x z}$ can be obtained directly using constitutive relation or by integrating equilibrium equation. In the present study, both the methods are used for TSDT-ZZ and the results are compared with 


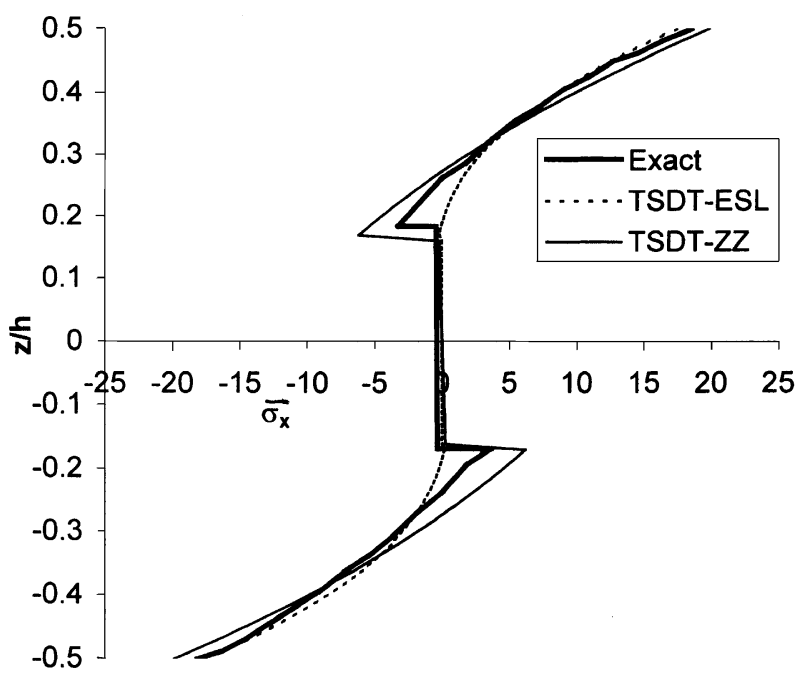

Fig. 4. Comparison of in-plane stress $\bar{\sigma}_{x}$ with exact results.

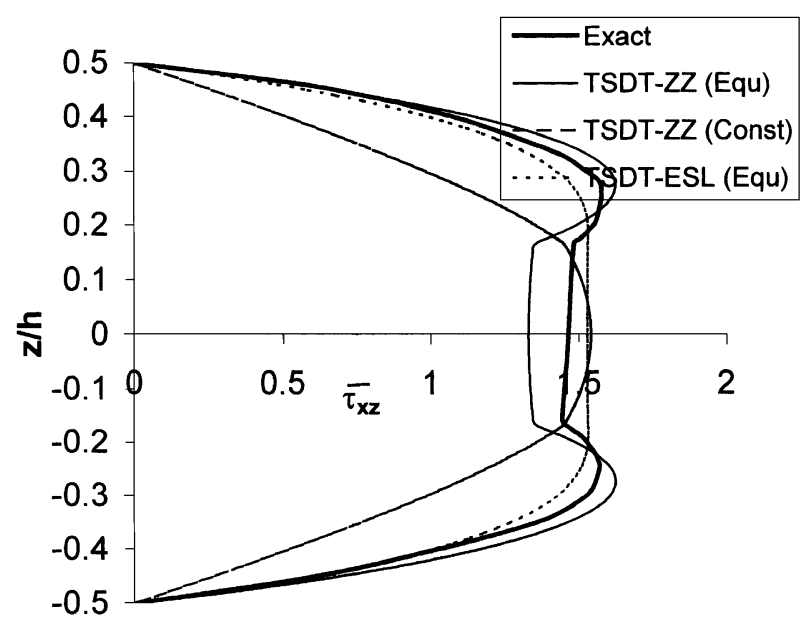

Fig. 5. Comparison of transverse shear stress $\bar{\tau}_{x z}$ with exact results.

the exact results and are shown in Fig. 5. In case of TSDT-ESL only equilibrium equation results are shown, knowing that it is discontinuous using constitutive relation and are not of much value. The trend of transverse shear stress obtained using TSDT-ZZ and equilibrium equation approach matches with that of exact results. The location of minimum and maximum stress are predicted well as compared to TSDT-ESL.

It can be noted that TSDT-ZZ requires one additional variable as compared to TSDT-ESL i.e. $u_{0}$. For symmetric laminates this term is zero in the case of TSDT-ESL and it can be further simplified to:

$u=-z \frac{\partial w_{0}}{\partial x}+\sin \frac{\pi z}{h} \eta_{x}$

$w=w_{0}$, $\gamma_{x z}=\frac{\pi}{h} \cos \frac{\pi z}{h} \eta_{x}$

In above equation it can be noted that single cosine term is able to give zero shear strain at top and bottom of the beam, whereas in polynomial type displacement model at least one more term will be required to satisfy this condition. This feature of TSDT makes it elegant and results in lesser number of stress resultants in governing equations.

\section{Concluding remarks}

In this paper, a new type of displacement function is presented. The displacement function uses trigonometric terms to represent the displacement across the thickness and able to capture the kink in displacement field in thick laminate. The displacement function satisfies displacement and transverse shear stress continuity at the interface. Zero shear stress condition at top and bottom of the beam is also satisfied. Results are also presented for a similar class of equivalent single layer beam theory. Results presented for transverse displacement, in-plane displacement, in-plane stress and transverse shear stress agree with the exact results. Comparison between zigzag and ESL theory of this class is also discussed.

\section{References}

[1] Lo KH, Christensen RM, Wu EM. A higher order theory of plate deformation, Part 1: Homogeneous plates. J Appl Mech 1977;44: 663-8.

[2] Lo KH, Christensen RM, Wu EM. A higher order theory of plate deformation, Part 2: Laminated plates. J Appl Mech 1977;44:66976.

[3] Reddy JN. A simple higher order theory for laminated composite plates. J Appl Mech 1984;51:745-52.

[4] Doong JL, Lee C, Fung CP. Vibration and stability of laminated plates based on a modified plate theory. J Sound Vib 1991;151 (2):193-201.

[5] Lee KH, Lin ZW, Chow ST. Bidirectional bending of laminated composite plates using an improved zigzag model. Compos Struct 1994;28:283-94.

[6] Xiaoyu Li, Dashin Liu. Zigzag theory for composite laminates. AIAA J 1995;33(6):1163-5.

[7] Stein M. Nonlinear theory of plates and shells including the effect of transverse shearing. AIAA J 1986;24(9):1537-44.

[8] Shimpi RP, Ghugal YM. A layerwise trigonometric shear deformation theory for two layered cross-ply laminated beams. J Reinforced Plastics Compos 1999;18:1516-43.

[9] Shimpi RP, Ainapure AV. A beam finite element based on layerwise trigonometric shear deformation theory. Compos Struct 2001;53(2):153-62.

[10] Pagano NJ. Exact solution for composite laminates in cylindrical bending. J Compos Mater 1969;3:398-410. 